\title{
Aplicación del modelo de control en espacios de estado a partir de las pérdidas totales obtenidas del porcentaje de carbono de la chapa de acero al silicio
}

\section{Application control model in state spaces from the total loss percentage obtained from the carbon silicon steel sheet}

\author{
María Gabriela Mago-Ramos \\ Ph. D. Ingeniería \\ Universidad ECCI \\ Bogotá, Colombia \\ mmagor@ecci.edu.co \\ Jhon Jairo Olaya-Flórez \\ Ph. D. Ciencias de los Materiales \\ Universidad Nacional de Colombia \\ Bogotá, Colombia \\ jjolayaf@gmail.com
}

\author{
Luis Vallés-Defendine \\ Ph. D. Ingeniería \\ Universidad de Carabobo \\ Valencia, Venezuela \\ le.vallesd@gmail.com \\ Christian Palomino-Naranjo \\ Ingeniero Mecánico \\ Universidad ECCI \\ Bogotá, Colombia \\ palomino.christian@ecci.edu.co
}

\begin{abstract}
Resumen- Esta investigación muestra los modelos de control en espacios de estado que representan un modelo matemático descrito mediante un conjunto de entradas, salidas y variables de estado relacionadas por ecuaciones diferenciales de primer orden, a partir de las pérdidas totales obtenidas del porcentaje de carbono de la chapa de acero al silicio, con la cual se fabrican los núcleos de los transformadores de distribución. El modelo propuesto permite evaluar las señales de corriente en el lado primario, secundario y de magnetización a partir de las pérdidas totales, ajustando el valor del coeficiente de acoplamiento de la chapa de acero. La aplicación de este traerá ventajas a las empresas que fabrican o reparan estos equipos, ya que podrán diseñar la chapa de acero silicio bajo alguna condición de falla versus valores nominales, también se puede presentar el modelo propuesto de forma canónica o natural: controlable, no observable y estable (CøE).
\end{abstract}

Palabras clave - chapa de acero al silicio, modelo de control en espacios de estado, pérdidas totales, porcentaje de carbono.

Abstract- This research presents the control models in state space representing a mathematical model described by a set of inputs, outputs and state variables related by first-order differential equations from the total percentage obtained carbon losses veneer silicon steel cores which distribution transformers are manufactured. The proposed model allows evaluating current signals in the primary, secondary and magnetizing side from total losses, adjusting the coupling coefficient of the steel sheet. Its application will bring benefits to companies that manufacture or repair this equipment, as they can design the silicon steel sheet under a fault condition versus nominal values, you can also submit the proposed model of canonical form or nature: controllable and not observable and stable (COE).

Keywords- silicon steel sheet, model of state control spaces, total losses, percentage of carbon.

\section{INTRODUCCIÓN}

En ingeniería de control, una representación de espacios de estados es un modelo matemático de un sistema físico descrito mediante un conjunto de entradas, salidas y variables de estado relacionadas por ecuaciones diferenciales de primer orden que se combinan en una ecuación diferencial matricial de primer orden. Para prescindir del número de entradas, salidas y estados, las variables son expresadas como vectores y las ecuaciones algebraicas se escriben en forma matricial (esto último solo puede hacerse cuando el sistema dinámico es lineal e invariante en el tiempo). La representación de espacios de estado (también conocida como aproximación en el dominio del tiempo) 
provee un modo compacto y conveniente de modelar y analizar sistemas con múltiples entradas y salidas. Con entradas y salidas tendríamos que escribir varias veces la transformada de laplace para procesar toda la información del sistema. A diferencia de la aproximación en el dominio de la frecuencia, el uso de la representación de espacios de estado no está limitado a sistemas con componentes lineales ni con condiciones iniciales iguales a cero. El espacio de estado se refiere al espacio de dimensiones cuyos ejes coordenados están formados por variables de estados. El estado del sistema puede ser representado como un vector dentro de ese espacio. La creación del conocimiento está basada en la aplicación de un Modelo de Control en Espacios de Estado inédito, a partir de las pérdidas totales obtenidas del porcentaje de carbono de la chapa de acero al silicio. En el 2014 el grupo de investigación liderado por Zhang R. [1], [2] también realiza trabajos novedosos en modelos de control en espacios de estado en forma predictiva para sistemas de control optimizando su rendimiento en forma parcial ante alguna perturbación, aplicando controles de velocidad, sin embargo, la característica innovadora es compartida en esta investigación por la aplicación para los transformadores utilizados como objeto estudio. Previamente, habían realizado un artículo para el control de temperatura de un equipo industrial [3], cuya condición operativa para ese modelo de control puede aplicarse en investigaciones similares para equipos del sistema eléctrico, lo que sin duda mejoraría la confiabilidad a los operadores del sistema, de tal manera que, puedan diseñarse sistemas multivariantes [4] que se desacoplen como un sistema MISO (única salida y múltiples entradas) incorporando una función de transferencia con análisis de frecuencia de la red. El transformador monofásico de poste, tiene una eficiencia o rendimiento que dependen tanto de la potencia de salida como de entrada, donde las pérdidas en el núcleo y en los devanados son consideradas, por lo tanto para esa condición, un método de bucle cerrado para perturbaciones desconocidas [5] que incluya un actuador parcial ante alguna condición de falla, (aunque las perturbaciones podrían traer errores) harían posible incluir en el modelo de espacio de estado propuesto, un controlador-observador en forma predictiva [6], más adelante se explicará que el modelo es controlable, no observable y estable, pero con esta modificación se haría predictivo para evaluar otras condiciones en estos equipos y sistemas eléctricos. Por otra parte, cuando se utilicen transformadores trifásicos se pueden aplicar modelos de control robusto que satisfagan la incertidumbre y el manejo de restricciones sin perder la estabilidad [7], desarrollando un mapeo de la respuesta a la condición de falla [8] sin dejar de lado el marco de la reciprocidad como si el transformador tuviera una zona muerta no lineal [9] y fuera inestable, esto permitiría la comprobación de las ecuaciones propuestas. Las condiciones ambientales pueden incluirse si se utiliza un modelo difuso que se linealice por tramos [10], cuyos algoritmos recursivos cuadrados permitan bondad de ajuste que derivarán en sistemas multivariables [11], [12]. Estas investigaciones permitirán sin duda alguna, el desarrollo de controles especiales para estos equipos [13] con lo cual se fortalecen los sistemas de protección y la probabilidad de ocurrencia de fallas en forma imprevista.

\section{MODELOS DE CONTROL EN ESPACIOS DE ESTADO}

Es un modelo matemático de un sistema físico descrito mediante un conjunto de entradas, salidas y variables de estado relacionadas por ecuaciones diferenciales de primer orden que se combinan en una ecuación diferencial matricial de primer orden. Para prescindir del número de entradas, salidas y estados, las variables son expresadas como vectores y las ecuaciones algebraicas se escriben en forma matricial (esto último solo puede hacerse cuando el sistema dinámico es lineal e invariante en el tiempo). [14]. El estado del sistema puede ser representado como un vector dentro de ese espacio.

\subsection{Variables de estado}

Las variables de estado son el subconjunto más pequeño de variables de un sistema que pueden representar su estado dinámico completo en un determinado instante. En la Fig. 1 se muestra un modelo de espacio de estado típico, teniendo en cuenta que estas variables de estado deben ser linealmente independientes. El número mínimo de variables de estado necesarias para representar un sistema dado, $n$, es normalmente igual al orden de la ecuación diferencial que define al sistema. 
Fig. 1. MODELO DE ESPACIO DE ESTADO TíPICO

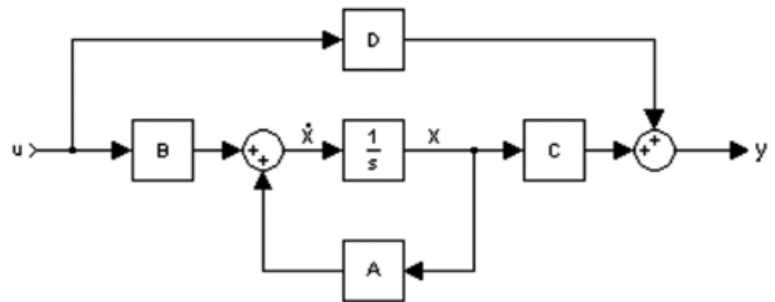

Fuente: http://es.wikipedia.org/wiki/Espacio_de_estados

\subsection{Espacios de estado:}

La relación existente entre variables de estado y entradas del sistema de acuerdo con la expresión matemática (1):

$$
x(t)=\psi\left(t, t o, x\left(t_{o}\right), u(\tau)\right)
$$

\subsubsection{Controlabilidad:}

Esta condición de estados implica que es posible, mediante entradas admisibles, dirigir los mismos desde cualquier valor inicial a cualquier valor final dentro de un intervalo de tiempo.

\subsubsection{Observabilidad:}

Es la medida de cuán correctamente los estados internos de un sistema pueden ser inferidos conociendo las salidas externas.

\subsection{Metodología}

Se trabaja con el circuito equivalente aproximado referido al lado secundario, además se ha simplificado la admitancia de magnetización. Las entradas son los voltajes $V_{1}$ y $V_{2}$, los estados: el cambio en la reactancia de magnetización y en la reactancia de la bobina del lado secundario, Fig. 2.

Fig. 2. CIRCUITO EQUIVALENTE DEL TRANSFORMADOR DE DISTRIBUCIÓN

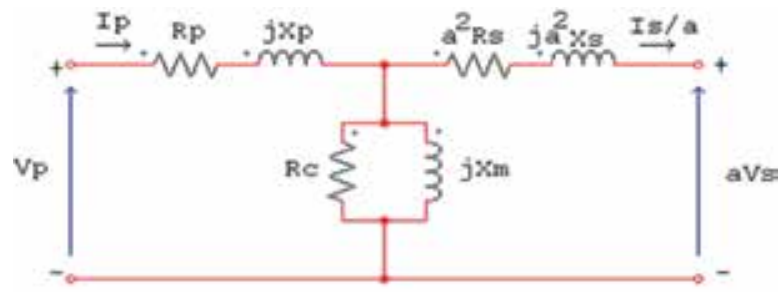

Fuente: http://dc388.4shared.com/doc/zT62xVEL/preview.html.

Donde:

$\mathrm{Vp}=$ voltaje del lado primario
Vs = voltaje del lado secundario

$\mathrm{a}=$ relación de espiras del transformador

$\mathrm{Rp}=$ resistencia del lado primario $(\Omega)$

$\mathrm{Xp}=$ reactancia del lado primario $(\Omega)$

Rs $=$ resistencia del lado secundario $(\Omega)$

$\mathrm{Xs}=$ reactancia del lado secundario $(\Omega)$

$\mathrm{Rc}=$ resistencia del núcleo o rama de magnetización $(\Omega)$

$\mathrm{Xm}=$ reactancia de magnetización $(\Omega)$

Ip $=$ corriente del lado primario (A)

Is $=$ corriente del lado secundario (A)

\subsubsection{Ecuaciones fundamentales}

corresponden a las entradas del modelo de estado, Voltaje $V_{1}$ y Voltajes $V_{1}$ y $V_{2}$ (2) y (3), respectivamente.

$$
\frac{V_{1(t)}}{a}=i_{0^{\prime}(t)} R_{E Q R}+L_{E Q M} \frac{d_{0^{\prime}(t)}}{d t} V_{1} \text { entrada de la }(2)
$$

$\frac{V_{1(t)}}{a}=i_{2(t)} R_{2}+L_{2} \frac{d_{i 2(t)}}{d t}+V_{2(t)} V_{1} y V_{2}$ entradas de la $(3)$

$\frac{d_{i^{0^{\prime}(t)}}}{d t}=\frac{\frac{V_{1(t)}}{a}-i_{0^{\prime}(t)} R_{E Q R}}{L_{E Q M}}(4)$ se despeja de la ecuación $(2)$

$\frac{d_{i 2(t)}}{d t}=\frac{\frac{V_{1(t)}}{a}-i_{2(t)} R_{2}-V_{2(t)}}{L_{2}}(5)$ se despeja de la ecuación $n(3)$

$X_{1}=i_{\sigma} y X_{2}=i_{2} \quad$ estados del mod elo propuesto

Se construye la ecuación en función de las entradas y estados, a partir de (4) y (5) indicadas anteriormente:

$$
\begin{aligned}
& \frac{d_{X 1(t)}}{d_{t}}=\frac{\frac{V_{1}}{a}-X_{1} R_{E Q R}}{L_{E Q M}}(6) \text { ecuación de entrada } V_{1} \\
& \text { en función del estado } X_{1} \\
& \frac{d_{X 2(t)}}{d_{t}}=\frac{\frac{V_{1}}{a}-X_{2} R_{2}-V_{2}}{L_{2}}(7) \text { ecuación de entrada } V_{1} y V_{2} \\
& \text { en función del estado } X_{2}
\end{aligned}
$$

\subsubsection{Modelo linealizado}

procedemos a linealizar el modelo de estado no lineal de la siguiente manera, obtener el modelo de estados con sus respectivas matrices: de estado, entrada, salida y transmisión directa (A, B $C$ y D). Para obtener respuesta del modelo utilizamos el programa MatlabSimulink@ y verificamos sus condiciones de funcionamiento (simulamos un proceso de operación con condiciones iniciales iguales a cero). 
$\left[\begin{array}{l}\Delta X_{1^{\prime}} \\ \Delta X_{2^{\prime}}\end{array}\right]=\left[\begin{array}{cc}-\frac{R_{E Q R}}{L_{E Q R}} & 0 \\ 0 & -\frac{R_{2}}{L_{2}}\end{array}\right]\left[\begin{array}{l}\Delta X_{1} \\ \Delta X_{2}\end{array}\right]+\left[\begin{array}{cc}\frac{V_{1}}{a} & 0 \\ \frac{L_{E Q M}}{L_{1}} & \\ \frac{a}{L_{2}} & -\frac{V_{2}}{L_{2}}\end{array}\right]\left[\begin{array}{l}\Delta L_{1} \\ \Delta L_{2}\end{array}\right]$ Matriz A

Matriz B

$$
Y_{t(t)}=\left[\begin{array}{ccc}
\frac{V_{1}}{\frac{a}{L_{E Q M}}} & \frac{V_{1}}{\frac{a}{L_{2}}} \\
0 & - & \frac{V_{2}}{L_{2}}
\end{array}\right]\left[\begin{array}{l}
X_{1} \\
X_{2}
\end{array}\right]
$$

Matriz C

Matriz D $=0$

$$
Z_{E Q}=\frac{\left|R_{C}\right| X_{m} \mid}{|Z|}<90^{\circ}-\varnothing=R_{E Q R}+j X_{E Q M}
$$

$$
\varnothing=\tan ^{-1}\left\{\frac{X_{m}}{R_{C}}\right\} ; V_{2}=N_{2} \frac{2 \pi f}{\sqrt{2}} \sqrt{\frac{10^{11} P_{t}}{10^{11} K_{H} f+2,2 f^{2} \Delta^{2}}}
$$

Las ecuaciones (8) y (9) corresponden a un transformador monofásico de $5 \mathrm{KVA}$, el cual fue obtenido a partir de los ensayos en vacío (sin carga) y cortocircuito, tal y como se indica a continuación:

Tap en 2, tensión del lado primario 13.800 Voltios, relación de espiras $\mathrm{a}=57.5$, donde $\mathrm{N}_{1}=$ 4715 y $\mathrm{N}_{2}=2 \times 41=82$. ( $\mathrm{N}_{1}$ son las espiras del lado primario y $\mathrm{N}_{2}$ las espiras del lado secundario).

\section{Resultado del ensayo en vacío (sin carga)}

$$
\begin{aligned}
& R_{C}(B T)=1309.0909 \Omega \\
& R_{C}(A T)=4328181.78812 \Omega \\
& X_{C}(B T)=348.84130 \Omega \\
& X_{C}(A T)=1153356.548112 \Omega
\end{aligned}
$$

\section{Resultado del ensayo en cortocircuito}

$$
\begin{aligned}
& X_{e q}(A T)=116.3575 \Omega \\
& X_{1}(A T)=X_{2}(A T)=58.1787 \Omega \\
& L_{1}(A T)=L_{2}(A T)=0.154323 \Omega \\
& R_{E Q}(A T)=822.7863 \Omega \\
& R_{1}(A T)\left(30^{\mathrm{o}}\right)=450.2 \Omega \\
& R_{1}(A T)\left(75^{\mathrm{o}}\right)=526.7935 \Omega \\
& R_{2}(A T)\left(75^{\mathrm{o}}\right)=295.992727 \Omega \\
& I_{n p}=0.3623 \text { Amp y } I_{n s}=20.8333 \text { Amp para } V_{2}= \\
& 240 \text { Volts. }
\end{aligned}
$$

Donde:

$R_{C}(B T)=$ resistencia del ensayo en vacío medida por el lado secundario $(\Omega)$

$R_{C}(A T)=$ resistencia del ensayo en vacío medida por el lado primario $(\Omega)$

$X_{1}(A T)=$ reactancia del lado primario medida por el lado primario $(\Omega)$

$X_{e q}(A T)=$ reactancia equivalente medida por el lado primario $(\Omega)$

$X_{2}(A T)=$ reactancia del lado secundario medida por el lado primario $(\Omega)$

$X_{C}(B T)=$ reactancia del ensayo en vacío medida por el lado secundario $(\Omega)$

$L_{2}(A T)=$ inductancia del lado secundario medida por el lado primario $(\Omega)$

$X_{C}(A T)=$ reactancia del ensayo en vacío medida por el lado primario $(\Omega)$

$L_{1}(A T)=$ inductancia del lado primario medida por el lado primario $(\Omega)$

$R_{E Q}(A T)=$ reactancia equivalente medida por el lado primario $(\Omega)$

$X_{C}(A T)=$ reactancia del ensayo en vacío medida por el lado primario $(\Omega)$

$R_{1}(A T)\left(30^{\circ}\right)=$ resistencia del lado primario medida por el lado primario a una temperatura de $30^{\circ}(\Omega)$

$R_{1}(A T)\left(75^{\circ}\right)=$ resistencia del lado primario medida por el lado primario a una temperatura de $75^{\circ}(\Omega)$

$R_{2}(A T)\left(75^{\circ}\right)=$ resistencia del lado secundario medida por el lado primario a una temperatura de $75^{\circ}(\Omega) \Omega$

$I_{n p}=$ corriente nominal del lado primario $(\mathrm{A})$

$I_{n s}=$ corriente nominal del lado secundario (A) $V_{2}=$ voltaje nominal del lado secundario $(\mathrm{V})$

\subsection{Pérdidas totales en el núcleo de los transformadores de distribución}

Las pérdidas totales en los transformadores de distribución dependen de las pérdidas por corrien- 
tes de Focault $(F)(10)$, Histéresis Magnética $(H)$ (11) y en el cobre, siendo estas últimas de rangos despreciables, finalmente la eq (12) correspondiente a las pérdidas totales. Los equipos que han estado operando bajo carga deben cumplir valores establecidos por la norma [15].

$$
\begin{gathered}
P_{F}=\frac{2,2 f^{2} \beta \max ^{2} \Delta^{2}}{10^{11}} \\
P_{H}=K_{H} . f . \beta \max ^{2}
\end{gathered}
$$

Pérdidas totales en el Núcleo $=P_{F}+P_{H}$

En la simulación se conecta a una carga resistiva de $11.52 \Omega$ correspondiente a los valores nominales de $\mathrm{V}_{2}$ en 240 Voltios e $\mathrm{I}_{2}$ en 20.83 Amp. Los valores de pérdidas totales obtenidos deben ser cambiados en el bloque correspondiente, al igual que el coeficiente de oscilación tal y como se indica a continuación en la tabla I:

TABLA I

PÉRDIDAS TOTALES VERSUS COEFICIENTE DE OSCILACIÓN

\begin{tabular}{|c|c|}
\hline Pérdidas totales $\left(\mathrm{P}_{\mathrm{t}}\right)$ & Coeficiente de oscilación $\left(\mathrm{K}_{\mathrm{H}}\right)$ \\
\hline 1.44 & 0.002498 \\
\hline 1.591 & 0.01199 \\
\hline 1.581 & 0.00274 \\
\hline 1.5940 & 0.00276 \\
\hline 1.5634 & 0.00271 \\
\hline 1.13 & 0.00203 \\
\hline 1.7577 & $(*)$ \\
\hline
\end{tabular}

Fuente: autores.

\subsubsection{Propiedades físicas del material}

Las propiedades físicas del material (chapa de acero al silicio, con la cual se fabrica el núcleo del transformador), como Resistencia eléctrica, Conductibilidad térmica, Saturación magnética, Fuerza coercitiva, Inducción remanente y Permeabilidad magnética. El análisis químico incluye dos mediciones y el valor promedio verificando los contenidos de hierro $(\mathrm{Fe})$, carbono $(\mathrm{C})$, manganeso $(\mathrm{Mn})$, fósforo $(\mathrm{P})$, azufre $(\mathrm{S})$, silicio $(\mathrm{Si})$, cobre $(\mathrm{Cu})$, niquel $(\mathrm{Ni})$, cromo $(\mathrm{Cr})$, vanadio $(\mathrm{V})$, molibdeno(Mo), wolframio o tusgteno $(\mathrm{W})$, cobalto (Co), titanio (Ti), estaño (Sn), aluminio (Al), niobio( $\mathrm{Nb})$, boro $(\mathrm{B})$, plomo (Pb), manganeso (Mg). El porcentaje de carbono obtenido y comparado con el valor promedio mostrado en la Tabla I es el referente utilizado para determinar las propiedades fisicas de las aleaciones de acero al silicio, entre las cuales se encuentran: la resistencia eléctrica y la fuerza coercitiva, la conductibilidad térmica, la inducción remanente y permeabilidad magnética. En la Fig. 3 se muestra la influencia del carbono en las propiedades fisicas del acero al silicio:

Fig. 3. INFLUENCIA DEL CARBONO EN LAS PROPIEDADES FÍSICAS DEL ACERO

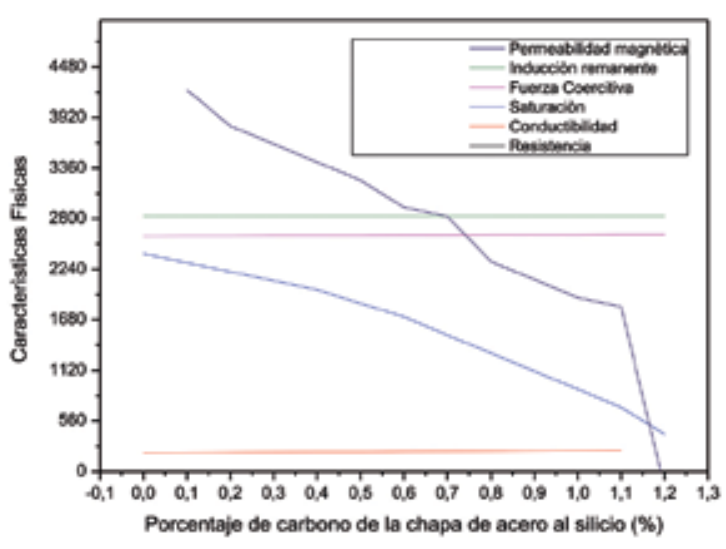

Fuente: autores.

La saturación magnética mostrada en la Fig. 4, es cualquier incremento posterior en un campo de magnetización externo $H$ no provoca un aumento en la magnetización del material, con la cual se obtiene el valor Bmáx requerido para determinar las pérdidas totales a través del cálculo del porcentaje de carbono, tablas II y III

\section{Fig. 4. SATURACIÓN MAGNÉTICA}

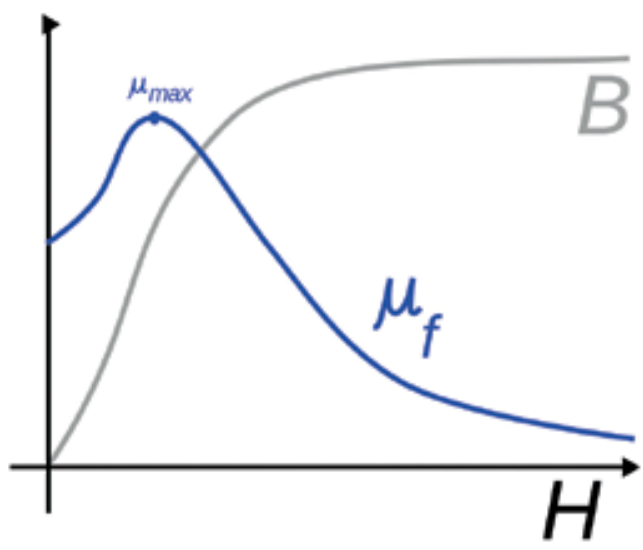

Fuente: http://es.m.wikipedia.org/wiki/Saturaciòn_magnètica. 
TABLA II

MUESTRAS DE EQUIPOS FALLADOS VERSUS \% DE CARBONO

\begin{tabular}{|c|c|c|}
\hline $\begin{array}{l}\text { Región de } \\
\text { procedencia }\end{array}$ & Tipo de falla & $\begin{array}{c}\text { Porcentaje de } \\
\text { carbono (\%) }\end{array}$ \\
\hline Antioquía & Térmica & 0.193 \\
\hline Antioquía & Descarga parcial & 0.069 \\
\hline Antioquía & Arco eléctrico & 0.010 \\
\hline Caldas & Térmica & 0.067 \\
\hline Caldas & Arco eléctrico & 0.040 \\
\hline Caldas & Arco eléctrico/térmica & 0.019 \\
\hline Caldas & Descarga parcial & 0.014 \\
\hline Cundinamarca & Térmica & 0.031 \\
\hline Bogotá & Arco eléctrico/térmica & 0.105 \\
\hline Bogotá & Arco eléctrico & 0.093 \\
\hline Bogotá & Descarga parcial & 0.101 \\
\hline Bogotá & Térmica & 0.1735 \\
\hline Pacífico & Térmica/Arco eléctrico & 0.131 \\
\hline Pacífico & Arco eléctrico & 0.002 \\
\hline Pacífico & Descarga parcial & 0.033 \\
\hline Patrón de referencia & & 0.033 \\
\hline
\end{tabular}

Fuente: autores

\subsubsection{Aplicación de métodos numéricos}

Se aplican métodos numéricos con el programa Matlab ${ }^{\odot}$. Construyendo una Recta de regresión $y=A x+B$ que mejor se ajusta en el sentido de los mínimos cuadrados a los $N$ datos $\left(X_{1}, Y_{1}\right), \ldots$ ., $(X n, Y n)[16]$.

$X=$ esta serie corresponde al porcentaje de carbono de la aleación.

$\mathrm{Y}=$ esta serie corresponde a la Saturación magnética.

2.4.2.1 Tipo de Falla: Térmica. Causa: Sobrecarga

A continuación se indica script realizado en el programa Matlab a fin de construir la recta de regresión. Cabe destacar que la respuesta del mé- todo numérico es dada en lenguaje diferente al español, por ejemplo Ans corresponde a la respuesta obtenida y viene predeterminada por el programa

$>$ X=[ $\begin{array}{llllllllll}0 & 0.1 & 0.193 & 0.2 & 0.3 & 0.4 & 0.5 & 0.6 & 0.7 & 0.8\end{array}$ $0.911 .11 .2]$

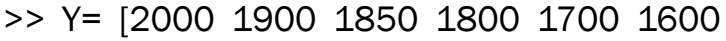
145013001100900700500300 0];

$>>$ Isline $(X, Y)$

Ans $=-1.6463 e+003$

$>>\operatorname{sum}(X)$ ans $=7.9910$

$>>\operatorname{sum}(\mathrm{Y})$ ans $=17100$

$>$ G $=((-7.9910 *-1.6463 e+003)+17100) /(14)$

$=2.1611 \mathrm{e}+003$

$>>y=(-1.6463 e+003 *(0.193))+2.1611 e+003$

$=1.8434 \mathrm{e}+003$ de Saturación magnética, corresponde a la densidad de flujo máximo, el cual será utilizado para determinar las pérdidas totales, tomando en consideración el peso del equipo, que para un transformador de capacidad 5 kVA es de 75_kg. A continuación en la Fig. 5 se muestra la saturación magnética obtenida versus porcentaje de carbono de la aleación que presentó la falla térmica debido a una sobrecarga:

Fig. 5. SATURACIÓN MAGNÉTICA VERSUS PORCENTAJE DE CARBONO

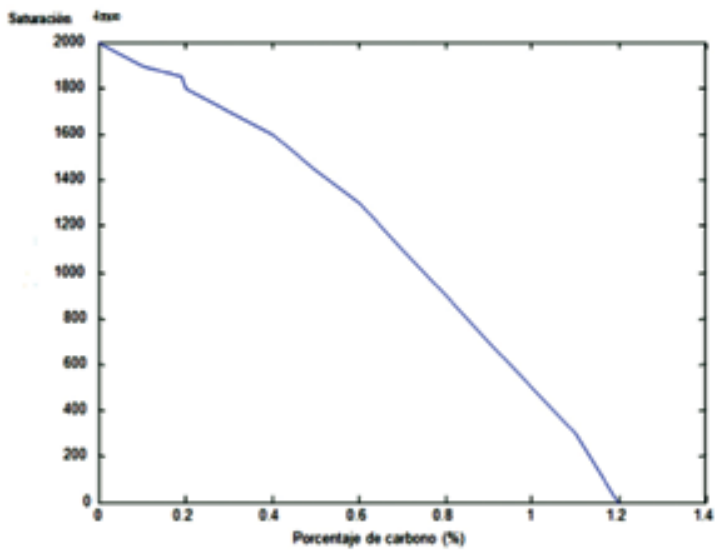

Fuente: autores.

A continuación en la Tabla III se muestran los resultados obtenidos de las pérdidas totales aplicando (10), (11) y (12), respectivamente. 
TABLA III

PÉRDIDAS TOTALES OBTENIDAS VERSUS \% DE CARBONO

\begin{tabular}{|l|l|c|}
\hline \multicolumn{1}{|c|}{$\begin{array}{c}\text { Región de } \\
\text { procedencia }\end{array}$} & \multicolumn{1}{|c|}{ Tipo de falla } & $\begin{array}{c}\text { Porcentaje de } \\
\text { carbono (\%) }\end{array}$ \\
\hline Antioquía & Térmica & 114.688596 \\
\hline Antioquía & Descarga parcial & 117.877042 \\
\hline Antioquía & Arco eléctrico & 119.368088 \\
\hline Caldas & Térmica & 117.880969 \\
\hline Caldas & Arco eléctrico & 118.586927 \\
\hline Caldas & Arco eléctrico/térmica & 119.133076 \\
\hline Caldas & Descarga parcial & 119.25154 \\
\hline Cundinamarca & Térmica & 139.324676 \\
\hline Bogotá & Arco eléctrico/térmica & 235.235044 \\
\hline Bogotá & Arco eléctrico & 117.257396 \\
\hline Bogotá & Arco eléctrico & 118.116709 \\
\hline Bogotá & Descarga parcial & 116.557365 \\
\hline Bogotá & Térmica & 115.111196 \\
\hline Pacífico & Térmica/Arco eléctrico & 158.569707 \\
\hline Pacífico & Arco eléctrico & 119.555869 \\
\hline Pacífico & Descarga parcial & 118.732706 \\
\hline Patrón de referencia & & 131.828066 \\
\hline Bures & \\
\hline
\end{tabular}

Fuente: autores.

\subsection{Esquema de modelo de estado utilizando Simulink@}

A continuación en la Fig. 6 se muestra el modelo de estado realizado en el espacio Matlab Simulink con el esquema que incluye las ecuaciones y gráficas de voltaje para el lado primario, secundario y rama de magnetización, que permiten a través del cambio en el coeficiente de acoplamiento variar las pérdidas totales del núcleo (Histéresis magnética y Corriente parásitas).

Fig. 6. MODELO DE ESTADO REALIZADO EN EL ESPACIO MATLAB SIMULINK

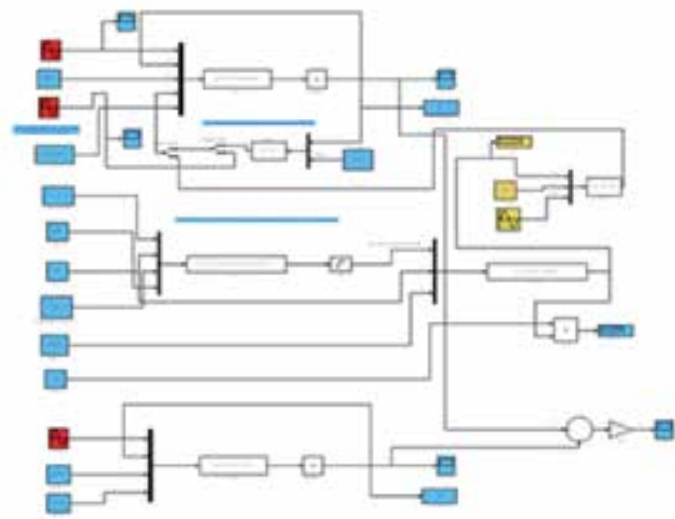

Fuente: autores.

\section{RESULTADOS}

Luego de aplicar el modelo de control en espacios de estado se obtienen las señales correspondientes en el lado secundario, primario y rama de magnetización, Figs. 7, 8 y 9.

Fig. 7. SEÑAL DE CORRIENTE EN EL LADO SECUNDARIO DEL TRANSFORMADOR

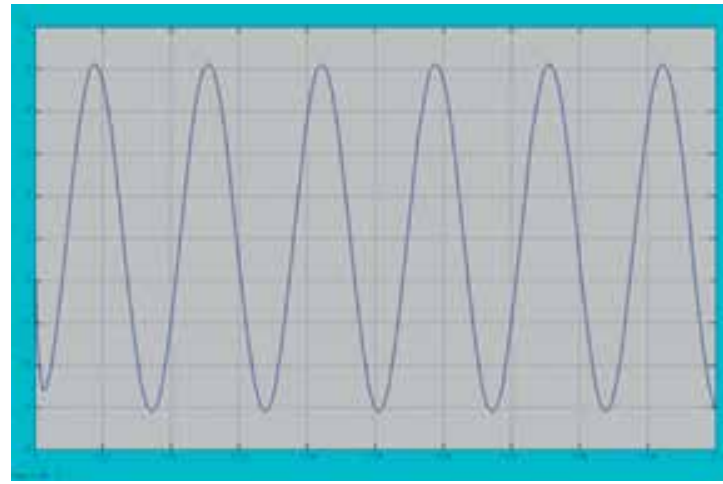

Fuente: autores.

Fig. 8. SEÑAL DE CORRIENTE EN EL LADO PRIMARIO DEL TRANSFORMADOR

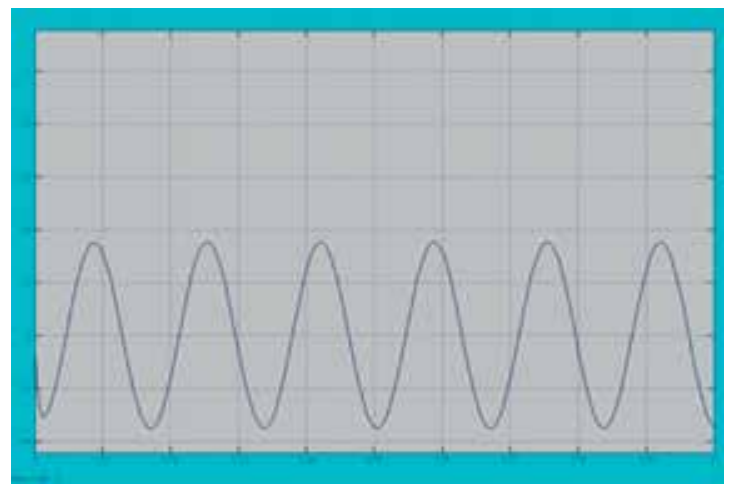

Fuente: autores.

Fig. 9. SEÑAL DE CORRIENTE EN LA RAMA DE MAGNETIZACIÓN DEL TRANSFORMADOR

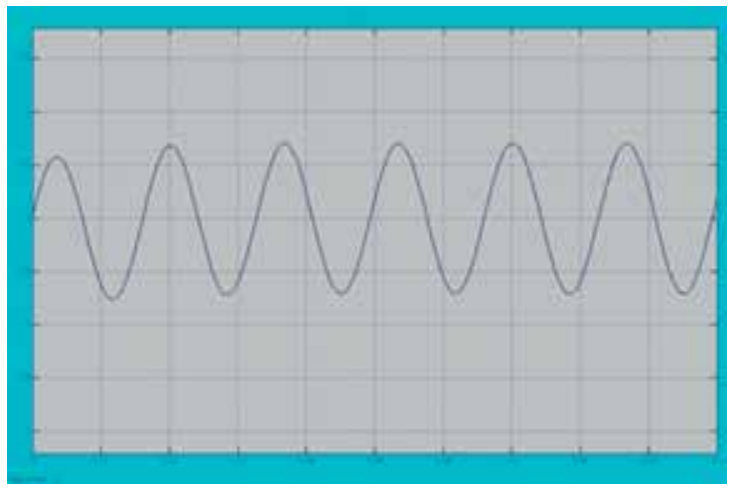

Fuente: autores. 


\subsection{Matrices de estado del modelo propuesto}

Utilizando el siguiente script del Matlab@ se obtienen las matrices de estado, entrada, salida y transmisión directa:

$$
\begin{aligned}
& \text { xo }=[20.31 ; 0.71] ; \\
& \text { ui }=[13800 ; 238.5] ; \\
& \text { yo }=[20.31 ; 0.71] ; \\
& \text { ix }=[] ; \\
& \text { iu }=[1 ; 1] ;
\end{aligned}
$$

$[\mathrm{x}, \mathrm{u}, \mathrm{y}, \mathrm{dx}] \quad=\quad$ trim('Proyecto_Mago_Entrada_ Salidar,xo,ui,yo,ix,iu)

$[A, B, C, D]=$ linmod('Proyecto_Mago_Entrada_ Salida', $\mathrm{X}$ )

Proyecto_Mago_Entrada_Salida = idss(A,B,C,D); idtf(Proyecto_Mago_Entrada_Salida)

\begin{tabular}{|c|c|c|c|}
\hline \multicolumn{2}{|c|}{ MATRIZ A: estado } & \multicolumn{3}{|c|}{ MATRIZ C: salida } \\
\hline-1918.1 & 0 & 1 & 0 \\
\hline 0 & -100.5 & 0 & 1 \\
\hline
\end{tabular}

MATRIZ B: entrada MATRIZ C: $\mathrm{D}=0$

Transmisión directa

\begin{tabular}{c|c}
372.6056 & 0 \\
0.0201 & 0
\end{tabular}

\subsubsection{Forma canónica controlable}

Esta condición de estados implica que es posible, mediante entradas admisibles, dirigir los mismos desde cualquier valor inicial a cualquier valor final dentro de un intervalo de tiempo, (13)

$$
\begin{gathered}
{\left[\begin{array}{c}
\dot{X_{1(t)}} \cdot \dot{\cdot} \\
X_{2(t)}
\end{array}\right]=\left[\begin{array}{cc}
-002020.0 & -192770.0 \\
000000.0 & 0
\end{array}\right]\left[\begin{array}{l}
x_{1(t)} \\
x_{2(t)}
\end{array}\right]+\left[\begin{array}{l}
1 \\
0
\end{array}\right] u(t)} \\
y(t)=\left[\begin{array}{ll}
0 & 0 \\
0 & 0
\end{array}\right]\left[\begin{array}{l}
x_{1(t)} \\
x_{2(t)}
\end{array}\right]+\left[\begin{array}{l}
0 \\
0
\end{array}\right] u(t)
\end{gathered}
$$

\subsubsection{Forma canónica observable}

Es la medida de cuán correctamente los estados internos de un sistema pueden ser inferidos conociendo las salidas externas, (14)

$$
\left[\begin{array}{c}
\dot{X_{1(t)}} \\
\cdot \\
X_{2(t)}
\end{array}\right]=\left[\begin{array}{cc}
-002020.0 & 000000.0 \\
-192770.0 & 0
\end{array}\right]\left[\begin{array}{l}
x_{1(t)} \\
x_{2(t)}
\end{array}\right]+\left[\begin{array}{l}
0 \\
0
\end{array}\right] u(t)
$$

$$
y(t)=\left[\begin{array}{ll}
1 & 0
\end{array}\right]\left[\begin{array}{l}
x_{1(t)} \\
x_{2(t)}
\end{array}\right]+\left[\begin{array}{l}
0 \\
0
\end{array}\right] u(t)
$$

\subsubsection{Función de transferencia}

esta respuesta caracteriza las relaciones de entrada-salida de componentes o de sistemas que se describen mediante ecuaciones diferenciales lineales invariantes con el tiempo, (15)

$\frac{Y(z)}{u(z)}=\frac{-0.0000+2535 i}{z-(0.0200+9.7722 i)}+\frac{-0.0000-2535 i}{z-(0.0002-9.7722 i)}$

\subsubsection{Matriz de estado del modelo propuesto}

La ecuación (16) es la ecuación de estado del sistema lineal e invariante con el tiempo, mientras que la (17) es la ecuación de salida para el mismo sistema

$$
\begin{gathered}
{\left[\begin{array}{c}
\dot{X}_{1} \\
\dot{X_{2}}
\end{array}\right]=\left[\begin{array}{ll}
0.0200 & +9.7722 i \\
0.0200 & -9.7722 i
\end{array}\right]\left[\begin{array}{l}
x_{1} \\
x_{2}
\end{array}\right]+\left[\begin{array}{l}
1 \\
1
\end{array}\right] u(z)} \\
Y(z)=[0.2535 i-0.2535 i]\left[\begin{array}{c}
\dot{X}_{1} \\
\cdot \\
X_{2}
\end{array}\right] \quad
\end{gathered}
$$

\section{DISCUSIÓN DE RESULTADOS}

El modelo en espacios de estado permite evaluar las señales de corriente en el lado primario, lado secundario y magnetización, a partir de las pérdidas totales, pero, ajustando el valor del coeficiente de oscilación. Esto traerá ventajas a las empresas que fabrican o reparan estos equipos, ya que podrán evaluarla chapa de acero al silicio bajo esta condición de falla versus condiciones nominales. Con respecto a las variables ambientales, específicamente la temperatura ambiente, aunque se ajusten los valores de las resistencias del lado y primario y secundario, "no es posible establecer un valor de temperatura mayor a $85^{\circ} \mathrm{C}$ o superior, dado que el punto de Curie es demasiado elevado", por los resultados obtenidos en las pruebas experimentales la chapa de acero al silicio "no pierde o cambia sus propiedades magnéticas", pero, se llena de óxidos o inclusiones debido a precipitados por alta temperatura que desmejoran la calidad térmica. La forma canóni- 
ca, esta viene a ser la forma más perfecta, pura, natural o simple en la cual se puede presentar un "modelo" matemático, que por norma matemática, debe ser presentado. El modelo de control en espacios de estado para el transformador de distribución, es controlable, no observable y estable.

\section{CONCLUSIONES}

En el caso de las pérdidas totales hay que ajustar el coeficiente de acoplamiento del material o $K_{H}$ que es una variable que depende del tipo de material, este ajuste hace que se revise el valor obtenido de la corriente nominal como punto de verificación para el modelo.

El sistema es controlable dado que el rango de la matriz es $\mathrm{n} \times \mathrm{n}$, "se dice que un modelo es controlable en el tiempo $t_{0}$ si se puede llevar de cualquier estado inicial $X_{t(0)}$ mediante un vector de control sin restricciones, en un intervalo de tiempo finito".

El modelo no es observable: "se dice que un modelo es observable en el tiempo $t_{0}$ si, el modelo en el estado $X_{(t 0)}$, es posible determinar este estado a partir de la observación de la salida durante un intervalo de tiempo finito".

"El modelo es estable ya que la parte real del número complejo en el módulo de los polos obtenidos son menores que uno, por lo tanto, están dentro del circulo unitario lo cual garantiza esta condición".

Estocásticamente el modelo desarrollado tiene una confiabilidad CØE (controlable, no observable y estable) de 76, 74\%.

\section{AGRADECIMIENTOS}

A la empresa TRANCECA en Venezuela y FYR Ingenieros en Colombia, de igual manera al Laboratorio de Tratamientos Térmicos de la Universidad Nacional de Colombia sede Bogotá y al CDCH (Centro de Desarrollo Científico y Humanístico) de la Universidad de Carabobo en Valencia, Venezuela.

\section{REFERENCIAS}

[1] R. Zhang, J. Lu, H. Qu, F. Gao, "State space model predictive fault-tolerant control for batch processes with partial actuator failure", Journal of Process Control, vol. 24, number 5. Information and Control Institute, Hangzhou Dianzi University, Hangzhou 310018, China and Department of Chemical and Biomolecular Engineering,
Hong Kong University of Science and Technology, Clear Water Bay, Kowloon, Hong Kong., 2014, pp. 613-620.

[2] R. Zhang, A. Zue, S. Wang, Z. Ren, "An improved model predictive control approach based on extended non-minimal state space formulation", Journal of Process Control, vol. 21, number 8 . Information and Control Institute, Hangzhou Dianzi University, Hangzhou 310018, China, National Key Laboratory of Industrial Control Technology, Institute of Cyber-Systems and Control, Zhejiang University, Hangzhou 310027, China, Department of Automation, Donghua University, Shanghai 200051, China, 2011, pp. 1183-1192.

[3] R. Zhang, A. Xue, F. Gao "Temperature control of industrial coke furnace using novel state space model predictive control", IEEE Transactions on Industrial Informatics, vol. 10, number 4. Control Institute, Hangzhou Dianzi University, Hangzhou, China, Chemical and Biomolecular Engineering Department, Hong Kong University of Science and Technology, Kowloon, Hong Kong, Information and Control Institute, Hangzhou Dianzi University, Hangzhou, China, 2014, 6881729, pp 2084-2092.

[4] R. Zhang, F. Gao, "State space model predictive control using partial decoupling and output weighting for improved model/plant mismatch performance," Industrial and Engineering Chemistry Research, vol. 52, number 2. Information and Control Institute, Hangzhou Dianzi University, Hangzhou 310018, China, Department of Chemical and Biomolecular Engineering, Hong Kong University of Science and Technology, Clear Water Bay, Kowloon, Hong Kong, National Key Laboratory of Industrial Control Technology, Institute of CyberSystems and Control, Zhejiang University, Hangzhou 310027, China, 2013, pp. 817-829.

[5] J. Tao, Y. Zhu, Q. Fan, "Improved state space model predictive control design for linear systems with partial actuator failure", Industrial and Engineering Chemistry Research, vol. 53, number 9. Ningbo Institute of Technology, Zhejiang University, Ningbo 315100, China, 2014, pp. 3578-3586.

[6] P. Tatjweski, "Disturbance modeling and state estimation for offset-free predictive control with state-space process models", International Journal of Applied Mathematics and Computer Science, vol. 24, number 2. Institute of Control and Computation Engineering, Warsaw University of Technology, ul. Nowowiejska 15/19, Warsaw, Poland, 2014, pp. 313-323.

[7] P. Falugi, S. Olaru, D. Dumur, "Multi-model predictive control based on LMI: From the adaptation of the state-space model to the analytic description of the controllaw", International Journal of Control, vol. 83, number 8. Department of Automatic Control, SUPELEC Systems Sciences (E3S), Gif sur Yvette 91192, France, 2010, pp.1548-1563.

[8] C. Shao, J. Sheng, "Stability analysis and control of linear periodic time-delay systems with state-space 
models based on semi-discretization", Proceedings of the 2012 UKACC International Conference on Control, Article number 6334729. Department of Mechanical Engineering, University of Michigan, Ann Arbor, MI, United States, Department of Automation, University of Science and Technology of China, Hefei, China, 2012, pp. $784-788$.

[9] Y-W. T-seng, Y-N. Wang, "Model following variable structure control design in reciprocal state space framework with dead-zone nonlinearity and lumped uncertainty", IEEE Conference on Industrial Electronics and Applications, Dept. of Electrical Engineering, I-Shou University, Kaohsiung, Taiwan, Wafer Test Factory, NXP Semiconductors Taiwan Ltd., Kaohsiung, Taiwan, ICIEA 2012 Singapore; 18 July 2012 through 20 July 2012; Category numberCFP1220A-CDR; Code 94705.

[10] W. Ya, C. Xin, W. Ming, H. Yong, "T-S fuzzy model based on time-delay state space for the control of burning through point", Conference Proceeding IEEE Computer Society, Article number 6639744. School of Information Science and Engineering, Central South University, Changsha 410083, China, Hunan Engineering Laboratory for Advanced Control and Intelligent Automation, Changsha 410083, China, 2013, Category numberCFP1340A-CDR; Code 101424, pp. 19401944.

[11] D. Honc, F. Düsek, "State-space constrained Model Predictive Control", Conference Proceeding , 27th European Conference on Modelling and Simulation, Code 105094, Department of Process Control, Faculty of Electrical Engineering and Informatics, University of Pardubice, nám. Čs. legií 565, 53210 Pardubice, Czech Republic, ECMS 2013, pp. 441-445.

[12] J. Zhang, "Improved nonminimal state space model predictive control for multivariable processes using a non-zero-pole decoupling formulation", Industrial and engineering chemistry research, vol. 52 number 13, State Key Laboratory of Industrial Control Technology, Institute of Cyber-Systems and Control, Zhejiang University, Hangzhou 310027, China, 2013, pp. 48744880 .

[13] Li. H. HongboZou, "Tuning of PI-PD controller using extended non-minimal state space model predictive control for the stabilized gasoline vapor pressure in a stabilized tower" Chemometrics and Intelligent Laboratory System., vol. 142. Information and Control Institute, Hangzhou Dianzi University, Hangzhou, China, 2015, pp. $1-8$

[14] K. Ogata, “Ingeniería de Control Moderna”, Editorial Prentice Hall. Quinta edición. México, 2010.

[15] Norma RA7-60. Valores de Pérdidas Equipos Bajo Condición de Carga. Disponible: http://www.epm.com.co/ site/Portals/0/Users/001/01/1/RA7-060.pdf. Consultado: 20-06-12 hora: 10:30 am.

[16] J. Mathews, K. Find, "Métodos Numéricos con Matlab". Editorial Prentice Hall. Tercera Edición. Madrid, España, 2005.

[17] E. STAFF DEL M.I.T. (1981). Circuitos Magnéticos y Transformadores. Editorial Reverte. Argentina.

[18] Forero., A (1999) Laboratorio de Metales. Universidad Nacional de Colombia. Facultad de Ingeniería. Bogotá, Colombia.

[19] Lajtin, Y (1983). "Metalografía y Tratamiento Térmico de los Metales".Tercera edición. ditorial MIR. Moscú. URSS.

[20] Smith, W. (2006). "Fundamentos de la Ciencia e Ingeniería de los Materiales". Editorial Mc Graw Hill. Cuartaedición. España. 